\title{
As formas de valoração e afrontamento na formação de professores (inicial) dos estudantes dos cursos de educação física
}

\section{Forms of valuation and afrontamento in teacher formation (initial) of the students of physical education}

\author{
${ }^{1}$ Felipe da Silva Triani \\ ${ }^{2}$ Cristina Novikoff
}

\section{RESUMO}

A pesquisa versa sobre as representações sociais acerca da formação inicial para estudantes do curso de Educação Física da Universidade denominada - UniEstudada. Ancora-se no projeto denominado "As representações sociais sobre formação de professores (inicial) para os professores dos cursos de pedagogia e de licenciaturas”, desenvolvido no Laboratório de Estudos e Pesquisas em Representações Sociais na/para Formação de Professores - LAGERES. O Objetivo é discutir as formas de enfrentamento que os estudantes estão adotando no seu agir e no pensar a formação de professores (inicial) diante das situações problema na graduação. O método de trabalho foi de natureza quanti-qualitativo, com abordagem mista. Neste artigo, apresenta-se a análise do Inventário de Valoração e Afrontamento - IVA (NOVIKOFF, 2006). Os resultados demonstraram que os discentes frente às situações-problema têm valoração como "ameaça” e "desafio". Nas estratégias de afrontamento, são centradas na "situação" para o grupo "Afetivo" e na "emoção” para o grupo "Apoio da Instituição de Ensino Superior”. Já a conduta motora é centrada na "situação” e "emoção” para os dois grupos. E o método de afrontamento é levemente evitativo e também passivo. A conclusão que o estudo obteve, é de que os estudantes estão lidando com as práticas acadêmicas de forma evitativa, com tendência maior à passividade.

Palavras-chave: Representações Sociais. Formação de Professores. Educação Física.

\section{ABSTRACT}

The research deals with the social representations of initial training for students of Physical Education at the University of Grande Rio - UniEstudada. Anchored in the project called "Social representations on teacher training (initial) for teachers of pedagogy courses and degrees", developed at the Laboratory of Studies and Research in Social Representations in / for Teacher Education - LAGERES. The goal is to discuss ways of coping that students are adopting in its acting and thinking teacher training (initial) problem situations before graduation. The method of work was both quantitative and qualitative, with mixed approach. In this article, we present the analysis of the Inventory Valuation and Confrontation - IAC (NOVIKOFF, 2006). The results showed that students face the problem situations have valuation as a "threat" and "challenge." In coping strategies are focused on the "situation" for the group "affective" and "emotion" to the group "Support of Higher Education Institution". Motor behavior is already focused on the "situation" and "emotion" for the two groups. And the method is slightly avoidant coping and also liability. The conclusion that the study found, is that students are giving to the academic practices so avoidant, with greater tendency to passivity.

Keywords: Social Representations. Teacher Training. Physical Education.

1 Aluno de Iniciação Científica - UNIGRANRIO

2 Docente do Mestrado Interdisciplinar em Letras e Ciências Humanas - UNIGRANRIO. Docente do Mestrado em Ensino em Saúde e Meio Ambiente - UniFOA. Coordenadora do Laboratório do Grupo de Estudos e Pesquisas em Representações Sociais na/para Formação de Professores - LAGERES 


\section{INTRODUÇÃO}

A Teoria de Moscovici (1979) acerca das representações sociais, atualmente, ganha espaço inigualável na academia. Neste sentido, a teoria moscoviciana vem provando seu fôlego para o duplo papel da pesquisa compreender e intervir na realidade. Em Gibbons e seus colaboradores (1994) encontra-se o respaldo a esta afirmativa da necessidade da ciência trabalhar num novo modo de produção do conhecimento situado num contexto de aplicabilidade. Assim, afirma-se com eles e outros (MORIN, 2004; SANTOS, 2003) que já não basta desenvolver pesquisas básicas - apenas de interesses cognitivos, mas articuladas a necessidade de resolver problemas práticos. Implica, portanto na produção do conhecimento transdisciplinar que num movimento relacional entre disciplinas se complementam na busca de soluções pragmáticas.

Nesta perspectiva o Laboratório do Grupo de Estudos e Pesquisas em Representações Sociais na/para Formação de Professores - LAGERES-UniEstudada, continuando o trabalho iniciado em 2004, com o grupo de estudos e pesquisas na/para as representações sociais, segue com pesquisas sobre formação de professores e suas representações sociais. Recentemente esta desenvolveu a pesquisa denominada "As Representações Sociais sobre Formação de Professores (Inicial) para os professores dos cursos de Pedagogia e de Licenciaturas”, com apoio do CNPq. Entre os trabalhos daí alavancados está a proposta de se trabalhar com estudos de graduandos e pós-graduandos, sendo, lato e stricto sensu. Trabalhos que seguem a linha acima descrita e de resultados satisfatórios, propiciando o desenvolvimento científico e tecnológico.

A apropriação da Teoria das Representações Sociais pela produção científica da Educação Física brasileira ainda é tímida. Em uma busca realizada no início deste estudo, no site da Coordenação de Aperfeiçoamento de Pessoal de Nível Superior - CAPES, disponibilizam-se resumos de teses e dissertações da pós-graduação brasileira. Nele, encontrou-se oito trabalhos entre dissertações e teses que foram publicadas entre os anos de 2000 e 2012, ou seja, uma baixa quantidade na ocorrência de estudos em representações sociais, entretanto, os que ali estavam sinalizaram uma maior consistência teórica ao longo dos anos, perspectivando uma melhora qualitativa nos estudos em representações sociais na Educação Física brasileira. Isto evidencia a apropriação de novas formas de olhar os fenômenos inseridos nesta disciplina, contribuindo para sua compreensão e, consequentemente, intervenção do profissional que atua nesta área.

Neste cenário, atualmente são desenvolvidas pesquisas a partir da iniciação científica que pense a forma de entender as representações sociais de formação inicial de discentes, ou seja, a "forma de conhecimento, socialmente elaborada e compartilhada, com um objetivo prático, e que contribui para a construção de uma realidade comum a um conjunto social” (JODELET, 2001, p.22), bem como os valores desses estudantes que norteiam tais representações sociais. Os dados poderão ser trabalhados para encontrar caminhos metodológicos que levem os estudantes a uma maior produtividade científica. Portanto, a pesquisa intitulada "as representações sociais sobre formação de professores (inicial) para os professores dos cursos de pedagogia e de licenciaturas", é o que norteou a presente pesquisa.

Neste contexto, o objetivo deste estudo foi em uma perspectiva prática compreender qual (is) a (s) forma (s) de enfrentamento que os estudantes estão adotando no seu agir e no pensar a formação de professores (inicial) diante das situações problema na graduação.

Sendo assim, a premissa é que a Teoria das Representações Sociais de Serge Moscovici (1979) permite compreender como os grupos sociais entendem determinado objeto e daí ter pistas de como melhorar esta compreensão e suas ações, acredita-se que estudando as representações sociais dos graduandos do Curso de Educação Física da UniEstudada, será possível compreender melhor seu impacto no enfrentamento das práticas acadêmicas. 


\section{METODOLOGIA}

A abordagem metodológica pretendida na pesquisa foi centrada na pragmática para se compreender como o discente do curso de Educação Física em formação se relaciona com o professor em atuação na graduação num processo de formação inicial, articulando-se ao de formação continuada dialeticamente. Para tal, inicia-se o trabalho pela compreensão das representações sociais sobre formação de professores, na educação superior. Os procedimentos, em síntese, para este estudo foram de abordagem quantitativa e qualitativa. A quantitativa foi empregada na escolha e aplicação do instrumento de pesquisa o questionário semiestruturado (CZAJA; JOHNNY, 1996) e o Inventário de Valorização e Afrontamento (CANO-VINDEL; MIGUEL-TOBAL, 1992). Já a abordagem qualitativa (CRESWELL, 2007, GÜNTHER, 2006, NOVIKOFF, 2010) foi delineada pelo estudo profundo do instrumento, seus resultados e devolutiva por meio de entrevistas do tipo focalizada (MERTON; KENDALL, 1979; BAUER; GASKELL, 2003). Nesta perspectiva onde quantidade e qualidade se complementam, o desenho foi utilizado como técnica projetiva, bem como, paramétricas e não paramétricas. O instrumento estandardizado foi utilizado para estudar as estratégias de enfrentamento utilizadas.

Os sujeitos da pesquisa foram graduandos do curso de Educação Física da Universidade denominada de UniEstudada. A escolha foi aleatória, portanto, a amostra foi de dois alunos de cada período dos cursos de licenciatura e bacharelado.

A delimitação desta pesquisa se deu inicialmente pela escolha da instituição de ensino de dependência administrativa privada no nível de Ensino Superior. A escolha da Instituição de Ensino Superior (IES) em questão foi feita por afinidade de linhas de pesquisa e interesse na temática. Portanto, fizeram parte da pesquisa os cursos de Educação Física de Licenciatura e Bacharelado da UniEstudada/RJ, no Município de Duque de Caxias na Baixada Fluminense.

O estudo foi aprovado pelo Comitê de Ética em Pesquisa da Universidade do Grande Rio - Prof. José de Souza Herdy (UNIGRANRIO), sob guarda do CAAE de número 11200412.2.0000.5283 e realizado com aprovação dos discentes dos cursos de Educação Física envolvidos após assinatura do termo de consentimento livre e esclarecido (TCLE).

\section{RESULTADOS E DISCUSSÃO}

O Gráfico 1 representa parte dos resultados obtidos via Inventário de Valoração e Afrontamento (adaptado por NOVIKOFF, 2006), instrumento utilizado na presente pesquisa. Porém, para o tratamento dos dados foi adotado a análise de conteúdo (BARDIN, 2009), utilizando o procedimento em "caixas", sendo estabelecido o sistema de categorias, onde os elementos, nesta pesquisa, foram repartidos em 5, representados no Gráfico 1, saber: Avaliação; Aluno; Apoio Institucional, Financeiro e de outros cursos; Afetividade e/ou Relação aluno-professor; Outro. Tais categorias foram criadas a partir da coleta de dados na entrevista, na qual o cada entrevistado descreveu um problema encontrado por ele na graduação e esse problema foi direcionado a uma categoria, como exemplifica o gráfico 1. 


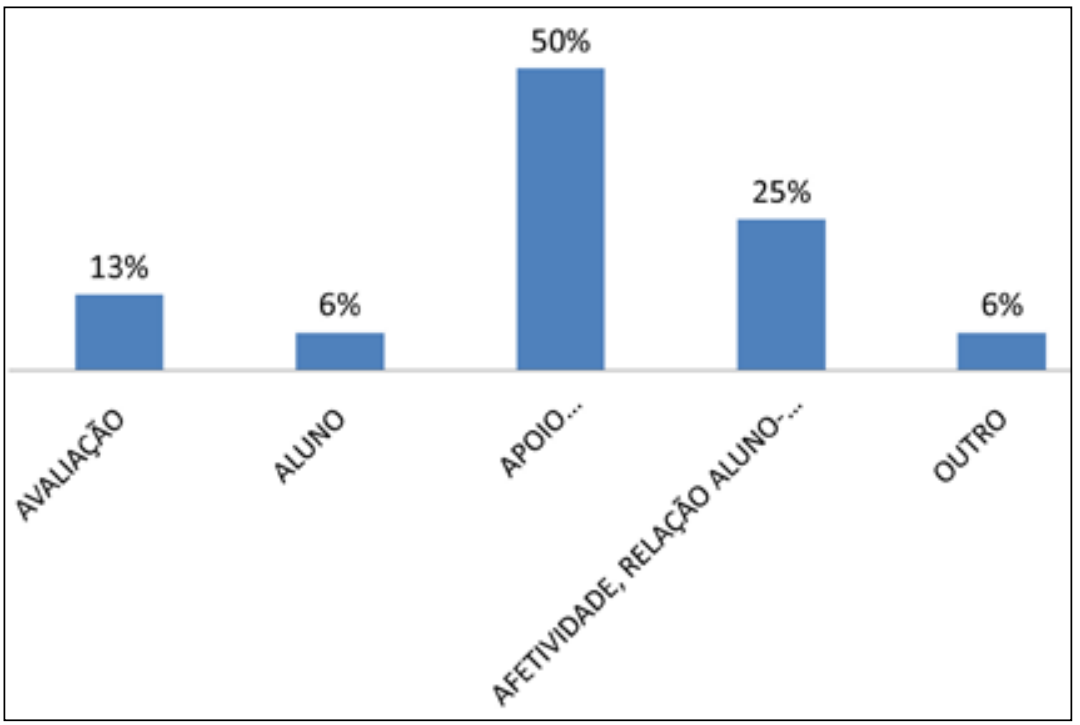

Gráfico 1: Os problemas levantados pelos discentes do curso de Educação Física.

É possível perceber que o primeiro elemento citado no ilustrativo acima é referente aos problemas na “Avaliação Acadêmica”, citado por 13\% dos sujeitos, em seguida é mencionado os próprios “Alunos”, como 6\% dos problemas encontrados no curso, depois surge com 50\% o "Apoio Institucional, Financeiro”. Na sequência, o quarto elemento demonstra que $25 \%$ dos entrevistados encontram um problema de "Afetividade e/ou relação aluno-professor". E, como um último elemento, denominado "Outro", referente a qualquer problema encontrado pelo sujeito da pesquisa que não se enquadra nos anteriores.

Neste contexto, a partir da interpretação gráfica, categorizamos os tópicos referentes aos elementos Apoio Institucional, Financeiro e de outros cursos, com $50 \%$ dos sujeitos denominando-os de "Apoio IES”, e o grupo Afetividade e/ou relação aluno-professor com 25\%, categorizado como "Afetivo”. Estes são tratados a diante, pois considerou-se que os outros elementos não possuem um percentual significativo para a pesquisa aqui tratada.

Nesta perspectiva, cada elemento está inserido em um contexto de 42 questões divididas em 9 categorias, que compõe o Inventário de Valoração e Afrontamento - IVA, instrumento utilizado na entrevista. Logo, foram analisados 18 gráficos representativos, sendo 9 referentes às categorias do grupo Apoio IES e os demais do grupo Afetivo.

Os 18 gráficos não são aqui apresentados, pois após análise detalhada de cada elemento dentro das categorias do IVA compilou-se os dados destes gráficos, gerando o somatório dos dados gerais obtidos como resultado de forma objetiva, no tabela 1. 
Tabela 1: Análise de categorias do IVA.

\begin{tabular}{|c|c|c|c|c|c|c|c|c|c|c|}
\hline \multirow[b]{2}{*}{$\begin{array}{c}\text { CATEGORIAS } \\
/ \\
\text { ITENS }\end{array}$} & \multicolumn{5}{|c|}{ AFETIVO } & \multicolumn{5}{|c|}{ APOIO IES } \\
\hline & 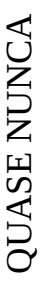 & 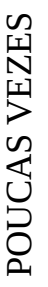 & 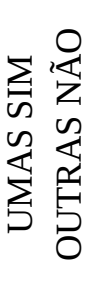 & 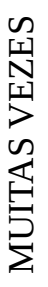 & 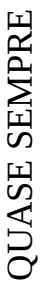 & 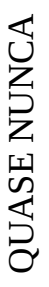 & 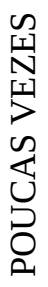 & 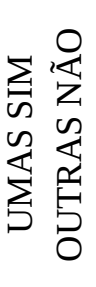 & 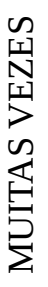 & 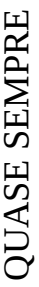 \\
\hline 1.VSA & 4 & 0 & 6 & 7 & 7 & 8 & 4 & 12 & 12 & 12 \\
\hline 2.VSD & 3 & 4 & 3 & 10 & 4 & 13 & 6 & 7 & 10 & 12 \\
\hline 3.VSI & 3 & 1 & 1 & 4 & 3 & 8 & 3 & 7 & 5 & 1 \\
\hline 4.ACS & 5 & 3 & 7 & 5 & 4 & 11 & 10 & 7 & 13 & 5 \\
\hline 5.ACE & 3 & 0 & 3 & 10 & 7 & 1 & 3 & 5 & 19 & 20 \\
\hline 6.AC-MS & 6 & 4 & 3 & 9 & 2 & 4 & 3 & 6 & 15 & 20 \\
\hline 7.AC-ME & 0 & 1 & 3 & 12 & 8 & 5 & 2 & 3 & 15 & 23 \\
\hline 8.AP & 1 & 2 & 4 & 1 & 0 & 11 & 2 & 1 & 2 & 0 \\
\hline 9. $\mathrm{AE}$ & 0 & 0 & 2 & 1 & 1 & 6 & 2 & $\mathbf{0}$ & 0 & 0 \\
\hline
\end{tabular}

Descrição dos itens: valoração da situação como ameaçadora (VSA); valoração da situação com um desafio (VSD); valoração da situação como irrelevante (VSI); afrontamento cognitivo centrado na situação (ACS); afrontamento cognitivo centrado na emoção (ACE); afrontamento de conduta motora centrada na situação (ACMS); afrontamento de conduta motora centrada na emoção (ACME); afrontamento passivo (AP); afrontamento evitativo (AE).

Através do resultado da soma dos itens, pode-se chegar ao grau de concordância e discordância de cada categoria, que são obtidos por meio da distribuição dos percentuais pelo total de respostas, conforme o Quadro 1:

Quadro 1: Descrição do Grau de Concordância e Discordância das Situações-Problema

\begin{tabular}{|c|c|c|}
\hline \% & GRAU DE CONCORDÂNCIA & GRAU DE DISCORDÂNCIA \\
\hline $0-15$ & baixo grau de concordância & baixo grau de discordância \\
\hline $16-35$ & leve grau de concordância & leve grau de discordância \\
\hline $36-55$ & intermediário grau de concordância & intermediário grau de discordância \\
\hline $56-75$ & bom grau de concordância & bom grau de discordância \\
\hline ACIMA DE 75 & alto grau de concordância & alto grau de discordância \\
\hline
\end{tabular}

O grau de concordância foi estabelecido em relação à sua tendência. Assim sendo, considerou-se o maior percentual indicado para assinalar o grau inicial, fazendo-se a soma dos itens de concordância ou discordância, indicadores da tendência do grupo.

Sendo assim, adotou-se uma tabela ilustrativa (Tabela 2), que aduz os dois elementos citados apresentados como situações-problema analisados no IVA. Logo, realizou-se novo somatório dos itens, a saber: 
Tabela 2: Comparação do grau de concordância e discordância das situações-problema

\begin{tabular}{|c|c|c|c|c|c|c|c|c|c|}
\hline \multirow{2}{*}{ GRUPOS } & \multicolumn{3}{|c|}{ VALORAÇÃO } & \multirow{2}{*}{$\begin{array}{c}\text { ESTRATÉGIA DE } \\
\text { AFRONTAMENTO } \\
\text { CENTRADA NA } \\
\text { SITUAÇÃO } \\
\%\end{array}$} & \multirow{2}{*}{$\begin{array}{c}\text { ESTRATÉGIA DE } \\
\text { AFRONTAMENTO } \\
\text { CENTRADA NA } \\
\text { EMOÇÃO } \\
\%\end{array}$} & \multirow{2}{*}{$\begin{array}{c}\text { FREQUÊNCIA DO } \\
\text { AFRONTAMENTO DE } \\
\text { CONDUTA-MOTORA } \\
\text { CENTRADA NA } \\
\text { SITUAÇÃO } \\
\%\end{array}$} & \multirow{2}{*}{$\begin{array}{c}\text { FREQUÊNCIA DO } \\
\text { AFRONTAMENTO DE } \\
\text { CONDUTA-MOTORA } \\
\text { CENTRADA NA } \\
\text { EMOÇÃO } \\
\%\end{array}$} & \multicolumn{2}{|c|}{$\begin{array}{c}\text { FORMA/MÉTODO DE } \\
\text { AFRONTAMENTO }\end{array}$} \\
\hline & $\begin{array}{l}\text { VSA } \\
\%\end{array}$ & $\begin{array}{c}\text { VSD } \\
\%\end{array}$ & $\begin{array}{c}\text { VSI } \\
\%\end{array}$ & & & & & $\begin{array}{c}\text { PASSIVO } \\
\%\end{array}$ & $\begin{array}{c}\text { EVITATIVO } \\
\%\end{array}$ \\
\hline AFETIVO & 100 & 100 & 50 & 100 & 96 & 100 & 100 & 33 & 17 \\
\hline APOIO IES & 100 & 100 & 50 & 96 & 100 & 100 & 100 & 33 & 17 \\
\hline
\end{tabular}

Comparando os resultados, observam-se diferenças entre as formas de valoração e afrontamento das situações-problema. Desta forma, evidenciam-se os graus de concordância e discordância entre as categorias.

Nesta mesma perspectiva, analisando o ilustrativo percebe-se que na categoria valoração das situações -problema os percentuais se igualam em graus de Ameaça e Desafio para os grupos.

Na sequência, o grau de concordância é alto para a Estratégia de Afrontamento Centrada na Situação e na Emoção, com maior destaque para Emoção para o grupo Apoio IES e na Situação para o Afetivo. Entretanto, a Frequência do Afrontamento de Conduta-Motora Centrada na Situação e na Emoção obteve alto grau de concordância e discordância, nos dois grupos. Na Forma/Método de Afrontamento Passivo apresenta leve grau de concordância para os dois grupos. Os grupos também apresentam leve grau de discordância do Método Evitativo.

Diante do exposto, implica dizer que os dois grupos de discentes, com situações-problema diferentes, têm a valoração como Ameaça e Desafio. Nas estratégias de afrontamento, são centradas na Situação para o grupo Afetivo e na Emoção para o grupo Apoio IES. Já a conduta motora é centrada na Situação e Emoção para os dois grupos. E a forma de afrontamento apresenta em ambos os grupos uma leve forma evitativa, com tendência à passividade. Noutras palavras, o sujeito evita o problema, com impulso a evitar enfrentar as dificuldades que ele mesmo assinala sendo um problema, adotando uma atitude apática ou indiferente, de aceitação do problema, sem discussão e naturalizando-o, uma vez que se sente ameaçado. Daí o desafio existe, mas não há enfrentamento cognitivo, apenas emotivo, com expressão de descaso, de apatia, de silêncio em sala de aula.

\section{CONCLUSÃO}

O presente estudo vem discutir na perspectiva prática, o lugar do conhecimento científico enquanto processo de experimentação, verificação, transcendência de fatos, análise crítica e comunicativa, exatidão e clareza (NOVIKOFF, 2010), articulado à Teoria de Moscovici (1979), como instrumento para se compreender e intervir na realidade, através de um contexto de aplicabilidade (GIBBONS et al, 1994). Deste modo, foi possível identificar as representações de estudantes do curso de Educação Física sobre a formação de professor.

Sendo as representações um fenômeno gerado por dados abstratos se elegeu como instrumento de coleta de dados, questionário e inventário, possibilitando o maior número possível de dados sobre as representações em estudo. Cabe destacar que a Teoria das Representações Sociais exige do pesquisador uma compreensão do lugar onde estas representações sociais são ancoradas. Daí ter sido aplicado os instrumentos de coleta de dados, no local de sua ancoragem - curso de Educação Física. E, para transformar os dados abstratos em concretos, foram realizadas entrevistas do tipo focalizadas (BAUER; GASKELL, 2003).

Ao concretizar as representações dos discentes dos cursos de Educação Física, foi possível compreender quais as formas de enfrentamento que os estudantes estão adotando no seu agir e pensar a formação de professores (inicial). Logo, identificou-se que quando frente a situações-problema os estudantes demonstraram uma valoração como "ameaça” e “desafio”; afrontam através da situação problema, usando de estratégias afetivas e, centradas na 
emoção; o grupo denominado de "institucionais", denota a conduta motora, para resolver seus problemas; diante de qualquer situação problema que se apresentou, o método de afrontar teve predominância "passiva”.

Em síntese, o estudo permitiu comprovar a premissa de que a Teoria das Representações Sociais pode possibilitar a compreensão de como os discentes dos Cursos de Educação Física da Universidade Estudada, enfrentam suas práticas acadêmicas. Nesta perspectiva, este estudo identificou que, na graduação os estudantes estão adotando seus métodos de enfrentar as situações problemas de forma evitativa, com tendência à passividade - um problema a ser discutido no colegiado deste curso estudado.

\section{REFERÊNCIAS BIBLIOGRÁFICAS}

BARDIN, L. Análise de Conteúdo. Lisboa, Portugal; Edições 70, LDA, 2009.

BAUER, Martin W.; GASKELL, G. Pesquisa qualitativa com texto, imagem e som: um manual prático. $2^{\mathrm{a} e d .}$ Pedrinho A. Guarechi (Trad). Petrópolis: Editora Vozes, 2003.

CANO-VINDEL, A.; MIGUEL-TOBAL, J.J. (1992). Inventario de valoración y afrontamiento. Madrid, Universidad Complutense de Madrid, Dpto. de Psicología Básica, mimeo. Adaptación argentina, Leibovich de Figueroa, N. B, (1999).

CRESWELL, J. W. Projeto de Pesquisa: métodos qualitativo, quantitativo e misto. Porto Alegre: Artmed, 2007.

CZAJA, Ronald and JOHNNY Blair. Designing Surveys: A Guide to Decisions and Procedures. Thousand Oaks, California: Pine Forge Press, 1996.

GIBBONS, M.; LIMOGES, C.; NOWOTNY, H.; SCHWARTZMAN, S.; SCOTT, P.; TROW, M. The new production of knowledge: the dynamics of science and research in contemporary societies, Londen, Sage, 1994.

GÜNTHER, Hartmut. Pesquisa Qualitativa Versus Pesquisa Quantitativa: Esta É a Questão? In Psicologia: Teoria e Pesquisa. mai-ago 2006, Vol. 22 n. 2, pp. 201-210.

JODELET, Denise. (org.). As representações sociais. Rio de Janeiro: Ed. Uerj, 2001.

MERTON, R.K.; KENDALL, P.L. Das fokussierte Interview. In: C. Hopf \& E. WingartenHeiner Legewie Interviewformen in der Forschung 21 (OrgS.). Qualitative Sozialforschung. Stuttgart: Klett-Cotta, 1979.

MORIN, Edgar. Os sete saberes necessários à educação do futuro. São Paulo. Ed. Cortez, Brasília, DF: UNESCO, 2004.

MOSCOVICI, Serge. A Representação Social sobre a Psicanálise. Rio de Janeiro: Zahar Editores, 1978.

Representações Sociais: investigações em psicologia social. Petrópolis: Ed.:Vozes, 2003.

NOVIKOFF, C. Dimensões Novikoff: um constructo para o ensino-aprendizado da pesquisa. In ROCHA, J.G. e NOVIKOFF, C. (orgs.). Desafios da práxis educacional à promoção humana na contemporaneidade. Rio de Janeiro: Espalhafato Comunicação, p. 211-242, 2010.

. As Representações sociais acerca de ensino superior para professores de graduação da área da saúde.

[Tese de doutorado]. São Paulo: Pontifícia Universidade Católica de São Paulo, 2006.

Os Caminhos da Construção Pedagógica: Instituindo o Ser Professor In: Escola Competente. 1 ed. Rio de Janeiro: Wak Editora, 2003.

SANTOS. Edméa Oliveira. Ambientes virtuais de aprendizagem: por autorias livre, plurais e gratuitas. In: Revista FAEBA, v.12, no. 18.2003. 